Article

\title{
An Anisotropic Scattering Analysis Method Based on the Statistical Properties of Multi-Angular SAR Images
}

\author{
Fei Teng ${ }^{1,2,3}$, Yun Lin ${ }^{4, *}$, Yanping Wang ${ }^{4}$, Wenjie Shen ${ }^{1,2,3}$, Shanshan Feng ${ }^{1,2,3}$ \\ and Wen Hong 1,3 \\ 1 Aerospace Information Research Institute, Chinese Academy of Sciences, Beijing 100094, China; \\ tengfei16@mails.ucas.ac.cn (F.T.); shenwenjie14@mails.ucas.ac.cn (W.S.); \\ fengshanshan17@mails.ucas.ac.cn (S.F.); whong@mail.ie.ac.cn (W.H.) \\ 2 University of Chinese Academy of Sciences, Beijing 101408, China \\ 3 Key Laboratory of Technology in Geospatial Information Processing and Application System, \\ Chinese Academy of Sciences, Beijing 100190, China \\ 4 School of Electronic Information Engineering, North China University of Technology, Beijing 100144, China; \\ wangyp@ncut.edu.cn \\ * Correspondence: ylin@ncut.edu.cn; Tel.: +86-135-8154-3995
}

Received: 10 June 2020; Accepted: 3 July 2020; Published: 5 July 2020

\begin{abstract}
The scatterings of many targets are aspect dependent, which is called anisotropy. Multi-angular synthetic aperture radar (SAR) is a suitable means of detecting this kind of anisotropic scattering behavior by viewing targets from different aspect angles. First, the statistical properties of anisotropic and isotropic scatterings are studied in this paper. X-band chamber circular SAR data are used. The result shows that isotropic scatterings have stable distributions in different aspect viewing angles while the distributions of anisotropic scatterings are various. Then the statistical properties of single polarization high-resolution multi-angular SAR images are modeled by different distributions. $G^{0}$ distribution performs best in all types of areas. An anisotropic scattering analysis method based on the multi-angular statistical properties is proposed. A likelihood ratio test based on $\mathrm{G}^{0}$ distribution is used to measure the anisotropy. Anisotropic scatterings can be discriminated from isotropic scatterings by thresholding. Besides, the scattering direction can also be estimated by our method. AHH polarization C-band circular SAR data are used to validate our method. The result of using $\mathrm{G}^{0}$ distribution is compared with the result of using Rayleigh distribution. The result of using $\mathrm{G}^{0}$ distribution is the better one.
\end{abstract}

Keywords: anisotropy; multi-angular; circular SAR; $\mathrm{G}^{0}$ distribution; likelihood ratio

\section{Introduction}

The scattering characteristic of the target is very important because it can be used for target detection, discrimination and classification. The scatterings of most man-made targets that people are interested in are aspect dependent, which is called anisotropy. With a common antenna aperture size, conventional synthetic aperture radar (SAR) working modes can obtain the scattering of the target within a few degrees due to the limited synthetic aperture length [1-3]. There are methods to detect the anisotropic scattering behavior using conventional SAR working modes [4,5]. Multi-angular SAR is a new SAR working mode [6-8]. It can obtain more complete scattering information by viewing the target under different aspect angles, so it is a suitable way to detect the anisotropic scattering behavior.

Anisotropic scattering analysis is very useful for SAR image interpretation so it has been widely researched in recent years. Zhao et al. extracted the multi-aspect scattering sensitivity feature 
which can characterize the anisotropic scattering $[9,10]$. Xue et al. analyzed the azimuthal changed polarimetric properties of targets [11]. Li et al. propose an anisotropic scattering detection method based on the multi-angular fully polarimetric signatures and constant false-alarm rate (CFAR) [12]. Ferro-Famil et al. used the likelihood ratio test to discriminate the non-stationary polarimetric behavior using fully polarimetric SAR data [5]. The likelihood ratio test is also used in SAR image change detection [13-15]. Most of the studies on anisotropic scattering are based on the fully polarimetric SAR data [16-18]. Actually, the amplitude of the single polarimetric SAR image includes anisotropic scattering information. However, limited synthetic aperture length make the amplitude variation not as sensitive and obvious as the polarimetric properties. If we use multi-angular SAR, the amplitude can better show the anisotropic scattering behavior of the target. Circular SAR (CSAR) is a kind of multi-angular SAR working mode. It can obtain the complete azimuthal scattering information of the target by $360^{\circ}$ observation [19-24]. C-band airborne CSAR data and X-band chamber CSAR data acquired by Aerospace Information Research Institute, Chinese Academy of Sciences (AIRCAS) are used to do the analysis and validate our method.

In this paper, we first analyze the statistical properties of anisotropic and isotropic scatterings. The result shows that the probability density functions (PDF) of the anisotropic scattering are various under different aspect angles, while the PDFs of isotropic scattering are relatively stable. This result means that anisotropic scatterings can be discriminated from isotropic scatterings by the different multi-angular statistical properties. To get a better understanding of the statistical property of the multi-angular SAR images, some frequently-used distributions are chosen to compare their fitting results. Some isotropic scattering areas and anisotropic areas are selected from the multi-angular SAR images. $G^{0}$ distribution has the best performance in all cases. Other distributions only perform well in some specific regions.

Then, an anisotropic scattering analysis method based on the statistical properties of multi-angular SAR images is proposed. The method uses the likelihood ratio test too. Differently from Ferro-Famil's method [5], we focus on anisotropic statistical properties of single polarimetric SAR images. Multi-angular SAR images are used to obtain more complete scattering information of the target and $G^{0}$ distribution is used to achieve a better effect in our method. The whole process of the anisotropic scattering analysis method is as follows. First, a conditional hypothesis representing anisotropic scattering and a null hypothesis representing isotropic scattering are given. Under the conditional hypothesis, the parameters of the PDF are assumed not all equal in different aspect angles. Under the null hypothesis, the parameters of the PDF are assumed all equal under all aspect angles. Second, the likelihood ratio is defined as the ratio of the conditional probability under these two hypotheses. Next, a sliding window is used to get the distributions from multi-angular SAR images, the likelihood ratio is calculated for each area and the value is assigned to the central pixel. Then, anisotropic scatterings can be discriminated from the isotropic scatterings by thresholding according to the likelihood ratio value. Finally, the scattering direction of the anisotropic scattering can be estimated by the similar likelihood ratio method using new hypotheses. Among the distribution models we used, $G^{0}$ distribution is the best fitted distribution for high resolution multi-angular SAR images. Therefore, the whole procedure for the $G^{0}$ approach is explained in detail in this paper. Rayleigh distribution is the scalar version of the Wishart distribution proposed in Ferro-Famil's method [5]. This paper briefly introduces the procedure of the Rayleigh distribution-based method for comparison.

The C-band circular SAR dataset mentioned before is used to generate the multi-angular SAR images. Any number of images under any angle can be obtained by sub-aperture segmentation. The method is tested using these multi-angular images. The result shows that anisotropic scatterings can be discriminated from isotropic scatterings by our method. The scattering direction estimated by the proposed method accords to that fact too. The result of using $\mathrm{G}^{0}$ distribution is better than using Rayleigh distribution. 


\section{Datasets}

Since multi-angular images are used from the very beginning of this paper, the datasets are introduced first. Circular SAR can provide $360^{\circ}$ SAR data and images so it can help the research on multi-angular SAR. Two circular SAR datasets are used in this paper. The first one is an X-band circular SAR dataset collected in a microwave anechoic chamber by us. Four canonical scatterers: a 160-mm dihedral, a 300-mm dihedral, a 200-mm top-hat and a 200-mm Luneberg ball are used in the experiment. The target is put on a turntable and the radar is put in a fixed position. The echo is collected every 0.2 degrees through the turntable rotation. In this way, the echo data of circular SAR can be obtained. The data is $\mathrm{HH}$ polarized with 1801 azimuth samples and 601 range samples. Figure 1 shows the optical images of the targets. These photos are screenshots of the CCTV in the chamber. The SAR image is obtained by coherently combining $360^{\circ}$-aperture-length data. The $360^{\circ}$ coherent complex SAR images of these targets are shown in Figure 2. The parameters are listed in Table 1.



(a) Small dihedral.

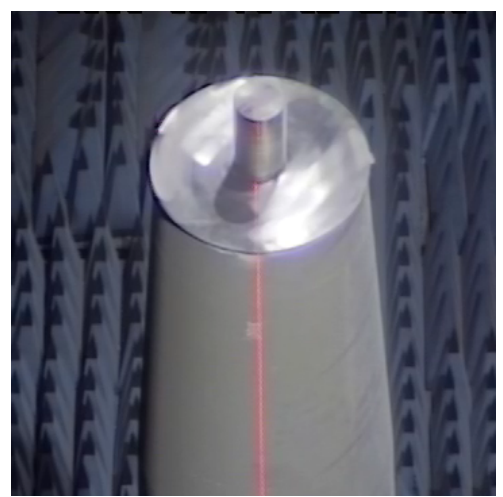

(c) Top-hat.

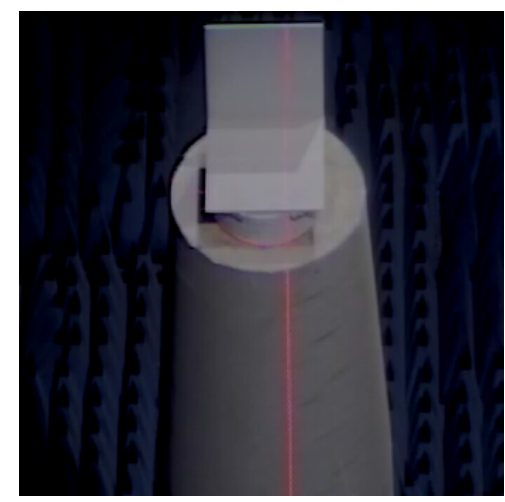

(b) Large dihedral.



(d) Luneberg ball.

Figure 1. Optical images of targets (d) Luneberg ball.
(a) Small dihedral.
(b) Large dihedral.
(c) Top-hat.

Table 1. Parameters of the X-band chamber circular SAR experiment.

\begin{tabular}{cc}
\hline Parameter & Description \\
\hline Carrier frequency & $15 \mathrm{GHz}$ \\
Bandwidth & $600 \mathrm{MHz}$ \\
Azimuthal sampling interval & $0.2^{\circ}$ \\
Look-down angle & $45^{\circ}$ \\
\hline
\end{tabular}




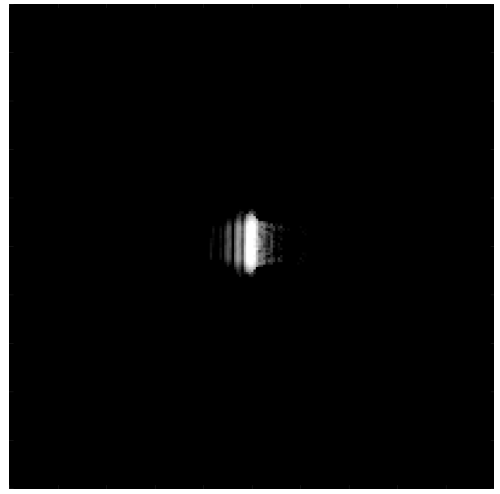

(a) Small dihedral.

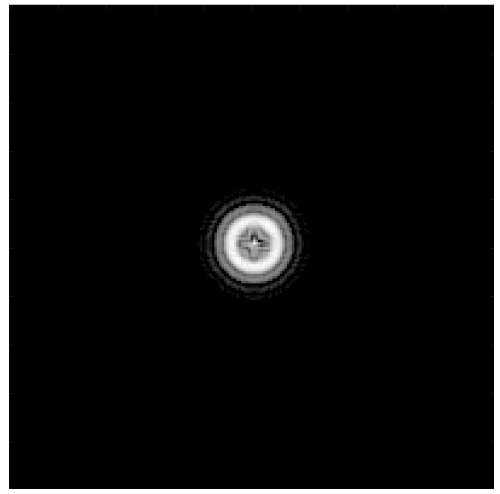

(c) Top-hat.

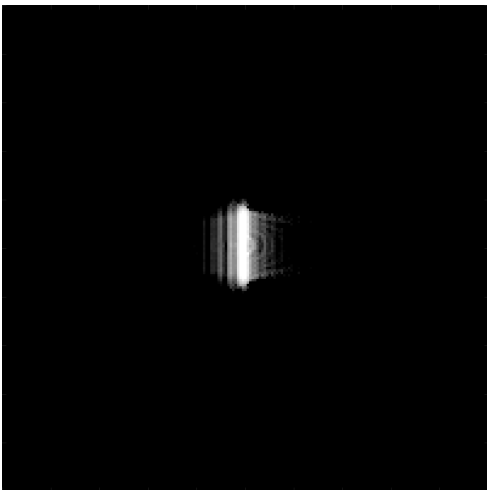

(b) Large dihedral.

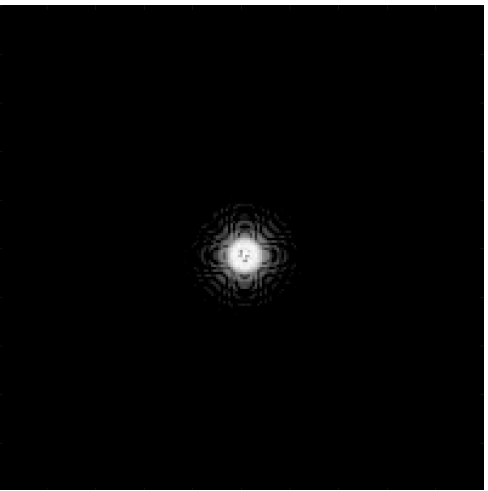

(d) Luneberg ball.

Figure 2. $360^{\circ}$ coherent complex SAR images of targets. (a) Small dihedral. (b) Large dihedral. (c) Top-hat. (d) Luneberg ball.

The second one is a C-band circular SAR dataset. It involves $\mathrm{HH}$ polarization too. It was acquired by the Aerospace Information Research Institute, Chinese Academy of Sciences (AIRCAS) in Zhuhai, Guangdong Province, China. Table 2 shows the experiment parameters. Figure 3 shows the optical image of the imaging scene and the flight trajectory. Figure 4 shows the $360^{\circ}$ coherent complex SAR image.

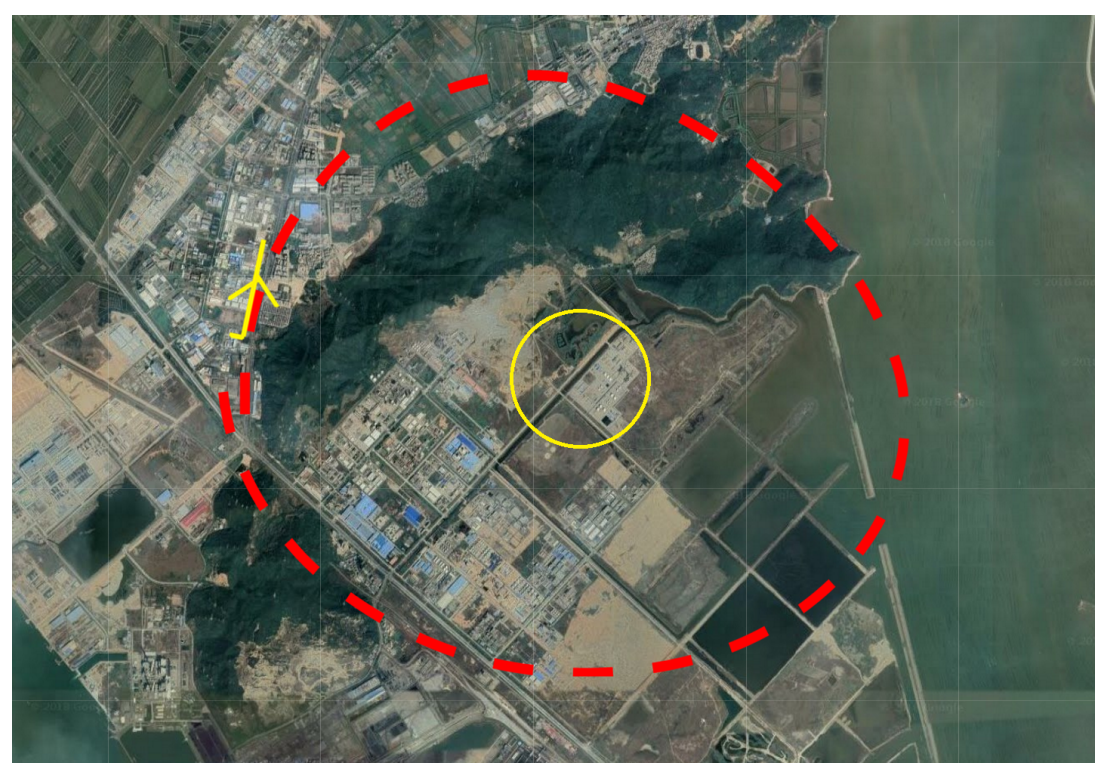

Figure 3. Optical image and the flight trajectory of the C-band circular SAR experiment. 
Table 2. Parameters of the C-band circular SAR experiment.

\begin{tabular}{cc}
\hline Parameter & Description \\
\hline Carrier frequency & $5.4 \mathrm{GHz}$ \\
Bandwidth & $560 \mathrm{MHz}$ \\
PRF & $2358 \mathrm{~Hz}$ \\
Look-down angle & $60^{\circ}$ \\
Flight height & $3 \mathrm{~km}$ \\
Flight Radius & $5 \mathrm{~km}$ \\
\hline
\end{tabular}

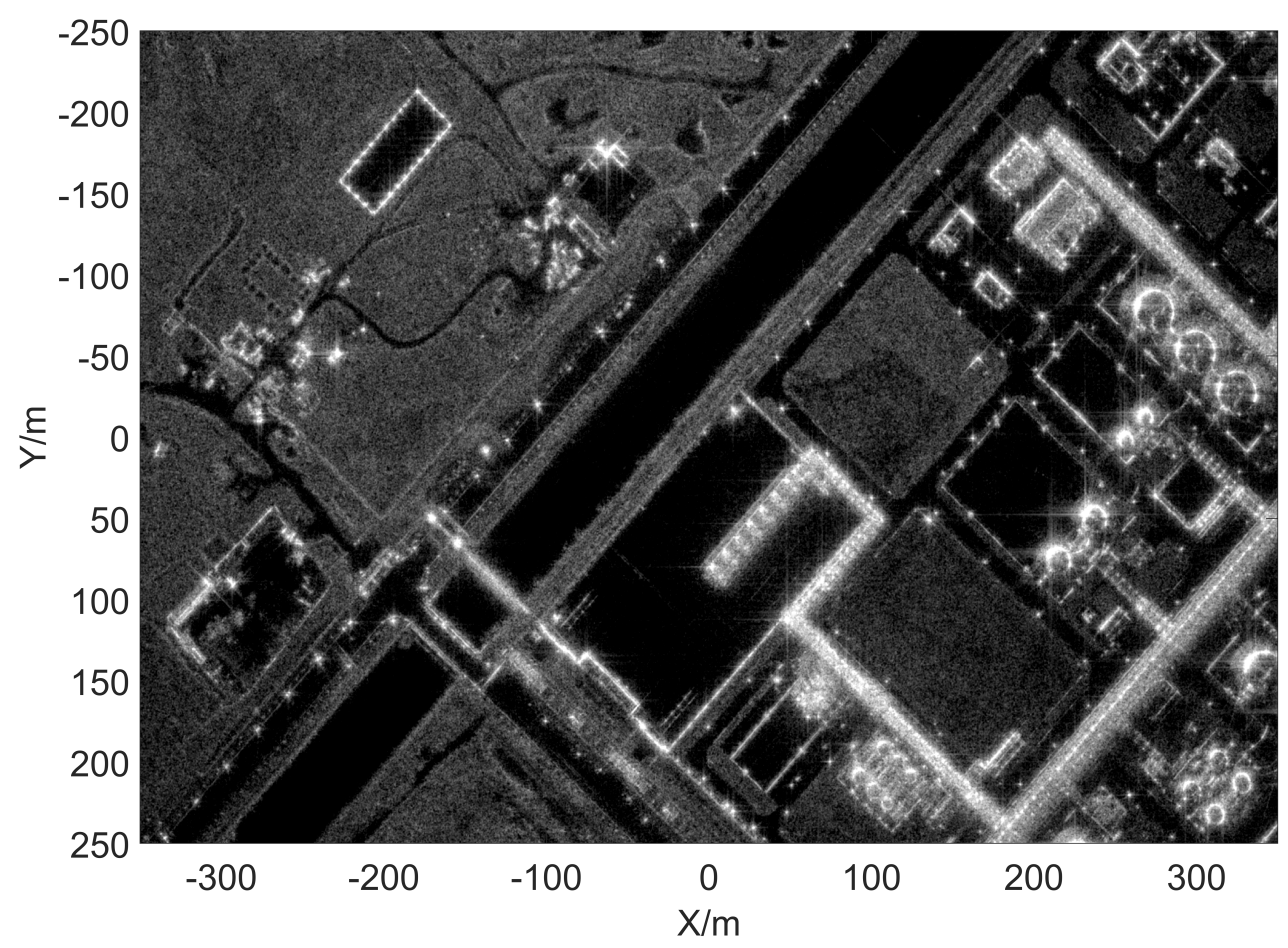

Figure $4.360^{\circ}$ coherent complex image of the C-band circular SAR.

\section{The Multi-Angular Statistical Property Analysis}

In this section, the multi-angular statistical property is analyzed. In the first subsection, the multi-angular images of four canonical scatterers are obtained by using the chamber data. Then, the PDFs of these targets in different angles are compared. The differences between them can be the approach to discriminating them from each other. In the second subsection, different areas are chosen from the multi-angular SAR images and modeled by several common distributions for SAR images. The fitting results are compared together.

\subsection{The Statistical Property of Anisotropic and Isotropic Scatterings}

The scattering is anisotropic if it is changing under different aspect angles. On the contrary, the scattering is isotropic if it is relatively stable under different aspect angles. In this paper, the scattering refers to the radar cross section (RCS) amplitude [25]; i.e., the amplitude of the SAR image. If the amplitude is various under different aspect angles, the statistical property of this area is supposed to be various too. There have been many experiments carried out in microwave anechoic chambers to study scattering characteristics, and they achieved good results [26,27]. Therefore, to remove as much influence of the environment and system as possible, we use the X-band circular SAR chamber data mentioned in Section 2 to observe the statistical property of the targets under different aspect angles. Four canonical scatterers are used. The small dihedral and the large dihedral are anisotropic targets. The top-hat and the Luneberg ball are isotropic targets. The multi-angular SAR images are 
generated by using back projection (BP) algorithm [28,29]. The full $360^{\circ}$ aperture is divided into 36 sub-apertures with $10^{\circ}$ gaps. The sub-aperture length is $3^{\circ}$ to make the azimuth resolution correspond to the range resolution. All the pixels in the target area are used to get the PDF. PDF curves of each sub-aperture images are obtained by calculating the densities of the pixel value. Figure 5 shows the PDFs of these four targets. As can be seen in the figures, PDFs of the top-hat and the Luneberg ball are basically stable under different aspect angles. As for the dihedrals, PDFs are various under different aspect angles. This demonstrates that the statistical properties of the anisotropic target are various under different aspect viewing angles. The statistical properties of the isotropic target are stable. This result can be used to discriminate anisotropic targets and isotropic targets from each other.

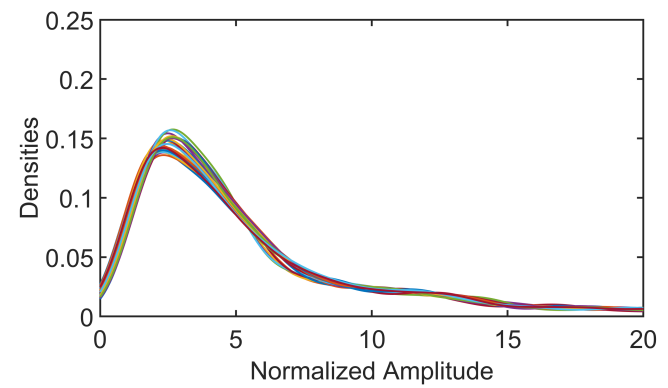

(a) Top-hat.

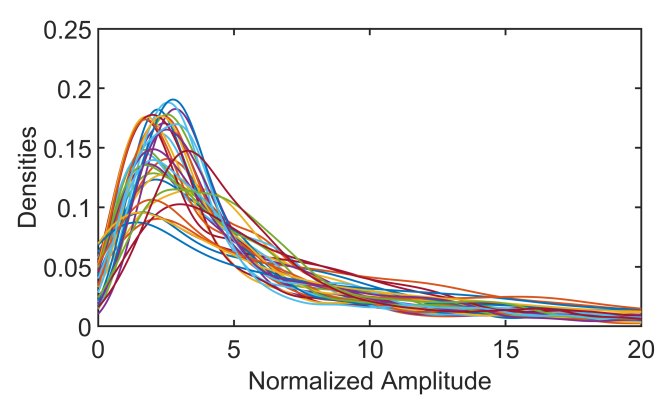

(c) Small dihedral.

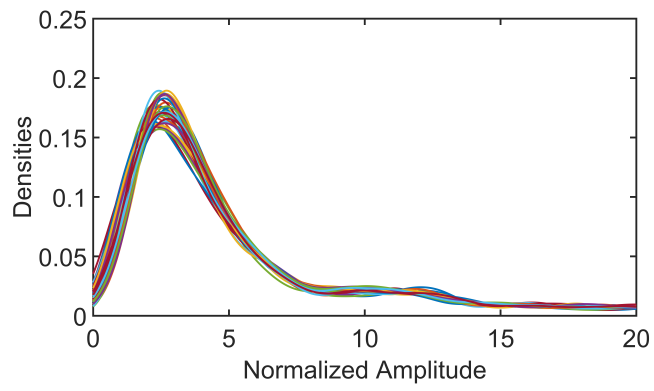

(b) Luneburg ball.

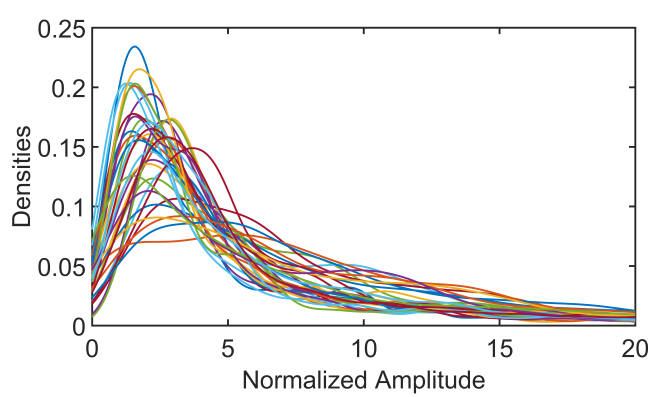

(d) Large dihedral.

Figure 5. The PDFs of targets under different aspect angles. (a) Top-hat. (b) Luneburg ball. (c) Small dihedral. (d) Large dihedral.

\subsection{The Statistical Model of the High-Resolution Multi-Angular SAR Images}

As mentioned before, the statistical properties can be used to discriminate the anisotropic scatterings and isotropic scatterings. To use the statistical properties of the multi-angular SAR images in further applications, the qualitative analysis of the shape of the PDF is not enough. The statistical distribution of multi-angular SAR images should be modeled. There are many statistical distribution models for conventional SAR images [30-33]. The models fall into three kinds: non-parametric models, parametric models and mixture distribution models. Parametric models are the most popular and widely researched. First, Rayleigh distribution and some other simple models are used to describe the homogeneous region in a low-resolution SAR image. With the development of the radar system and the occurring of new SAR working mode, distribution models should adapt to high resolution SAR images and heterogeneous areas. Therefore, distributions such as $\mathrm{K}$ distribution and $\mathrm{G}^{0}$ distribution are proposed. These distributions can model the extremely heterogeneous areas in high resolution SAR images. However, the parameter estimations for these kinds of distribution are more difficult than for simpler distributions.

Four different areas were chosen from the C-band circular SAR sub-aperture images and they were used to do the fitting. These areas include both anisotropic and isotropic areas. Isotropic areas were cut from the lawn and the concrete floor. Anisotropic areas were cut from a building and a 
storage tank. The sub-aperture length was $5^{\circ}$ to make the azimuth resolution correspond to the range resolution. The image cuts contained $100 \times 100$ pixels. This size is appropriate to include the entire targets. In this way, the complete statistical property of different targets can be obtained. Figure 6 shows the sub-aperture images of the chosen areas.

The non-linear least squares method is used to estimate the parameters while fitting the curve. The non-linear least squares method fits the model with a linear distribution and estimates the parameters by iteration. This is a mature and popular method of non-linear model fitting. There are many toolboxes can be used directly. Therefore, the details of the non-linear least squares method are not explained in this paper. The adjusted R-square and the root mean square error (RMSE) are used to measure the goodness-of-fit $[34,35]$. They are both frequently used evaluating indicator. The R-square is calculated by

$$
R^{2}=1-\frac{\sum(y-\widehat{y})^{2}}{\sum(y-\bar{y})^{2}}
$$

where $y$ is the actual value of the data, $\widehat{y}$ is the prediction value of the data and $\bar{y}$ is the mean value of the data. In order to get an absolute evaluation indicator, the number of parameters should be considered. Therefore, the adjusted R-square is proposed [36]. It is calculated by

$$
R_{a d j}^{2}=1-\left(1-R^{2}\right) \frac{(n-1)}{(n-k)}
$$

where $n$ is the number of the data and $k$ is the number of parameters. For adjusted R-square, the closer that the value is to 1 , the more similar the data and the distribution are. The RMSE value is calculated by

$$
R M S E=\sqrt{\sum(y-\bar{y})^{2}}
$$

Then different distributions are used to fit the data. We choose some most popular distributions: Rayleigh distribution [37], lognormal distribution [38], Weibull distribution [39], K distribution [40] and $\mathrm{G}^{0}$ distribution [41]. Figure 7 shows the fitting results of these four areas.

The closer the value to 0 , the greater the similarity is. The RMSE value is used as a supplement indicator in cases of the same adjusted R-square values. The adjusted R-square values of the fitting results are listed in Table 3. The RMSE values of the fitting results are listed in Table 4. As can be seen in the tables, $G^{0}$ distribution has the best performance in all areas. Other distributions only perform well in specific areas and perform badly in other areas. Therefore, $G^{0}$ distribution can describe homogeneous and heterogeneous; anisotropic and isotropic; and man-made and natural areas from the multi-angular SAR image.

Table 3. The adjusted R-square values of the fitting results.

\begin{tabular}{ccccc}
\hline Adjusted R-Square & Lawn & Concrete Floor & Building & Storage Tank \\
\hline Rayleigh & 0.9977 & 0.9542 & 0.7120 & 0.8305 \\
Lognormal & 0.9732 & 0.9767 & 0.9758 & 0.9857 \\
Weibull & 0.9983 & 0.9793 & 0.9314 & 0.9655 \\
K & 0.9989 & 0.9835 & 0.9501 & 0.9792 \\
$G^{0}$ & 0.9989 & 0.9907 & 0.9908 & 0.9959 \\
\hline
\end{tabular}




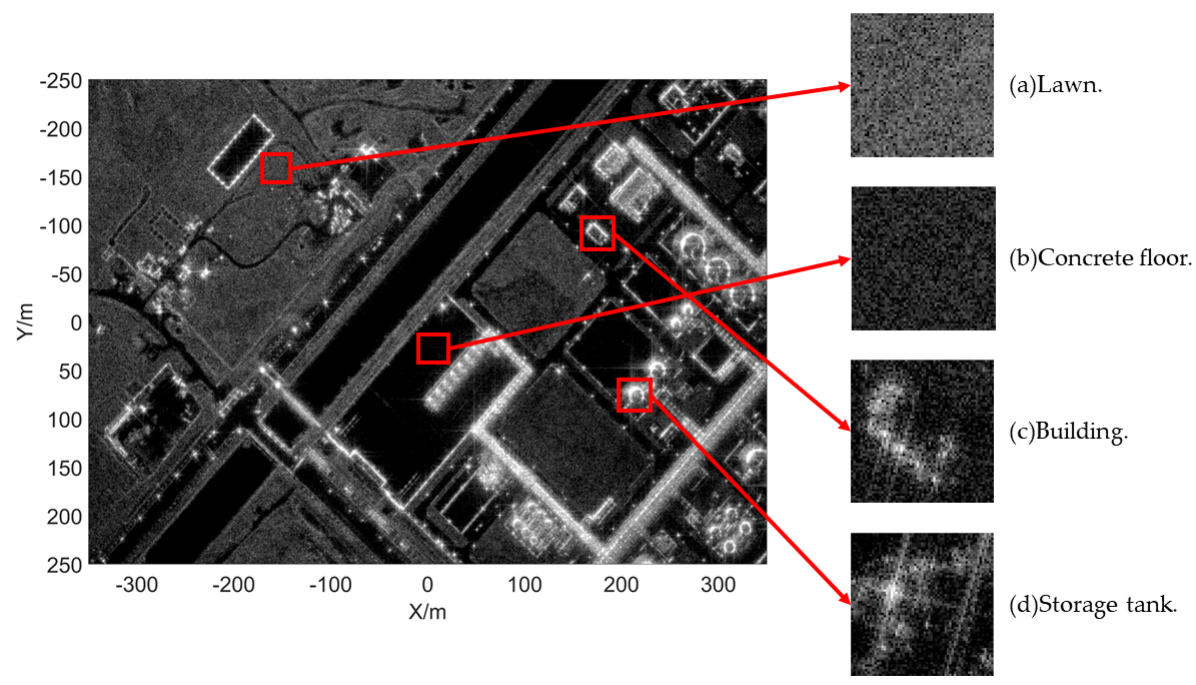

Figure 6. The sub-aperture images of chosen areas. (a) Lawn. (b) Concrete floor. (c) Building. (d) Storage tank.

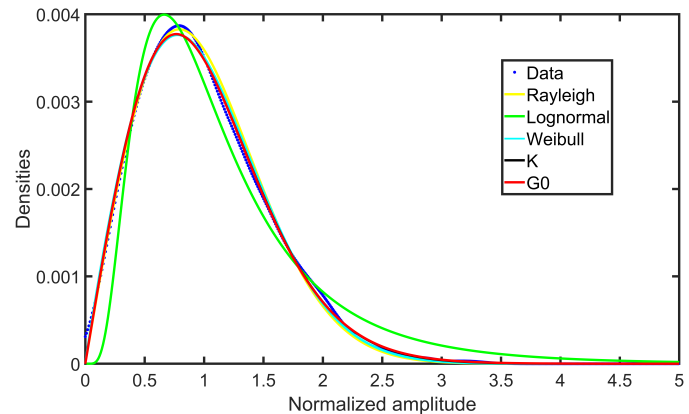

(a) Lawn.

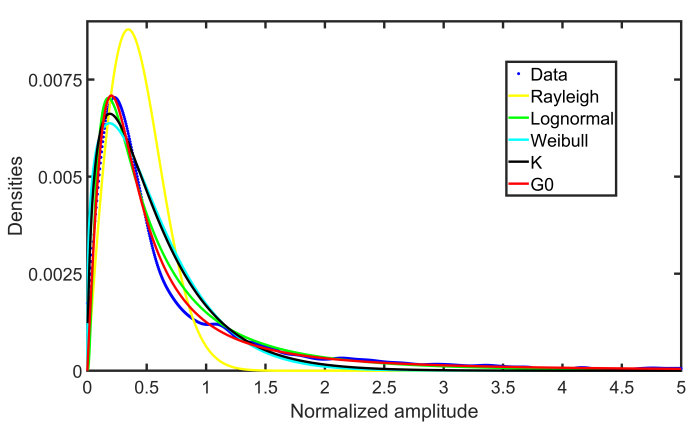

(c) Building.

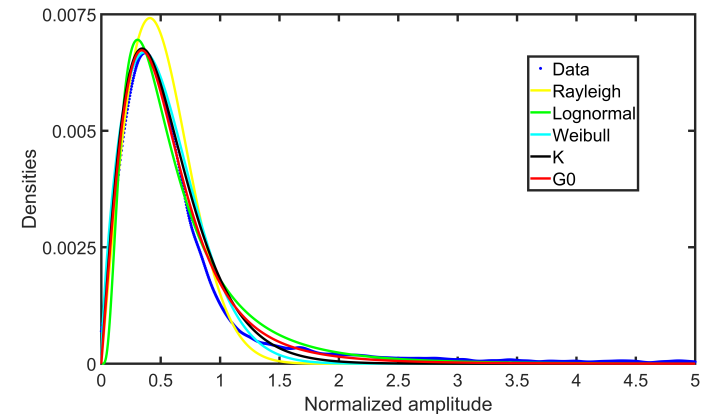

(b) Concrete floor.

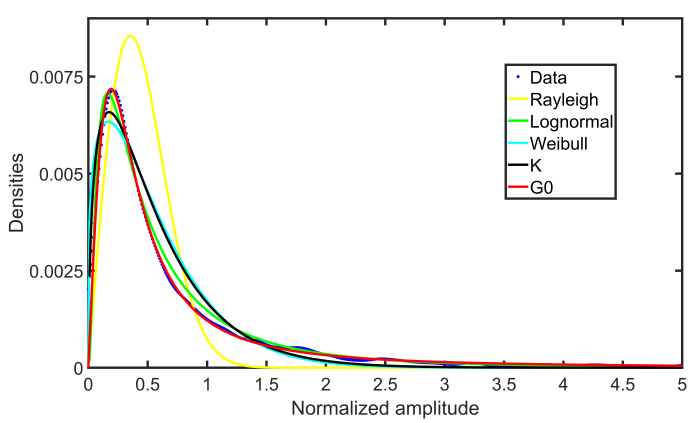

(d) Storage tank.

Figure 7. The fitting result of the chosen areas.

(a) Lawn. (b) Concrete floor.

(c) Building. (d) Storage tank.

Table 4. The RMSE values of the fitting results.

\begin{tabular}{ccccc}
\hline RMSE & Lawn & Concrete floor & Building & Storage Tank \\
\hline Rayleigh & 0.0100 & 0.0766 & 0.1364 & 0.0971 \\
Lognormal & 0.0344 & 0.0528 & 0.0395 & 0.0282 \\
Weibull & 0.0087 & 0.0547 & 0.0665 & 0.0439 \\
K & 0.0070 & 0.0460 & 0.0567 & 0.0340 \\
$\mathrm{G}^{0}$ & 0.0070 & 0.0345 & 0.0244 & 0.0150 \\
\hline
\end{tabular}




\section{Methodology}

In this section, the proposed anisotropic scattering analysis method is introduced in the first subsection. Then a method used for comparison is briefly introduced in the second subsection.

\subsection{Anisotropic Scattering Analysis Method}

The statistical properties of anisotropic scattering are various under different aspect angles. If we can measure the difference between these multi-angular statistical properties, anisotropic scatterings can be discriminated from the isotropic scatterings and the degree of anisotropy can also be described. The likelihood ratio test is widely used in SAR images' change detection. It is also used for anisotropic scattering detection on fully polarimetric SAR data [5]. It can detect the change of the parameters of the statistical distribution model. For multi-angular SAR images, it can also be used to detect the change caused by the scattering anisotropy. As shown in Section 3.2, $\mathrm{G}^{0}$ distribution can best describe the high-resolution multi-angular SAR images. Therefore, an anisotropic scattering analysis method based on the likelihood ratio test using $\mathrm{G}^{0}$ distribution is proposed in this paper.

The likelihood ratio is defined as the ratio of the conditional probability of accepting the alternative hypothesis and the null hypothesis.

$$
\lambda=\frac{P\left(X \mid H_{1}\right)}{P\left(X \mid H_{0}\right)}
$$

The value of the likelihood ratio refers to how many times more likely the data are under the alternative hypothesis than the null hypothesis. We give the binary hypothesis representing the anisotropic scattering and the isotropic scattering perspectively like this

$$
\begin{aligned}
& H_{0}: \sigma_{1}=\sigma_{2}=\cdots=\sigma_{N}=\sigma_{0} . \\
& H_{1}: \sigma_{1}, \sigma_{2}, \cdots, \sigma_{N} \text { are not all equal. }
\end{aligned}
$$

$N$ is the number of the multi-angular SAR images so there are $N$ parameters of the distribution under different angles. $\sigma$ is the parameter of the distribution of the multi-angular SAR image. $\sigma_{0}$ is a constant. For the null hypothesis, the parameters of the statistical property model are all the same under different aspect angles. That refers to the isotropic scattering. For the alternative hypothesis, the parameters of the statistical property model are not all the same. That refers to the anisotropic scattering. Thus, under the conditions in our method, the likelihood ratio expresses how likely the scattering is to be anisotropic over being isotropic. A higher value means the scattering is more anisotropic.

The whole procedure of the method is divided into five steps; the flowchart is shown in Figure 8. The first step is sub-aperture image generation. The second step is obtaining the distribution by the sliding window. The third step is likelihood ratio calculation. Next is thresholding and discrimination. Finally is the scattering direction estimation. The details of each step will be explained in following parts. 


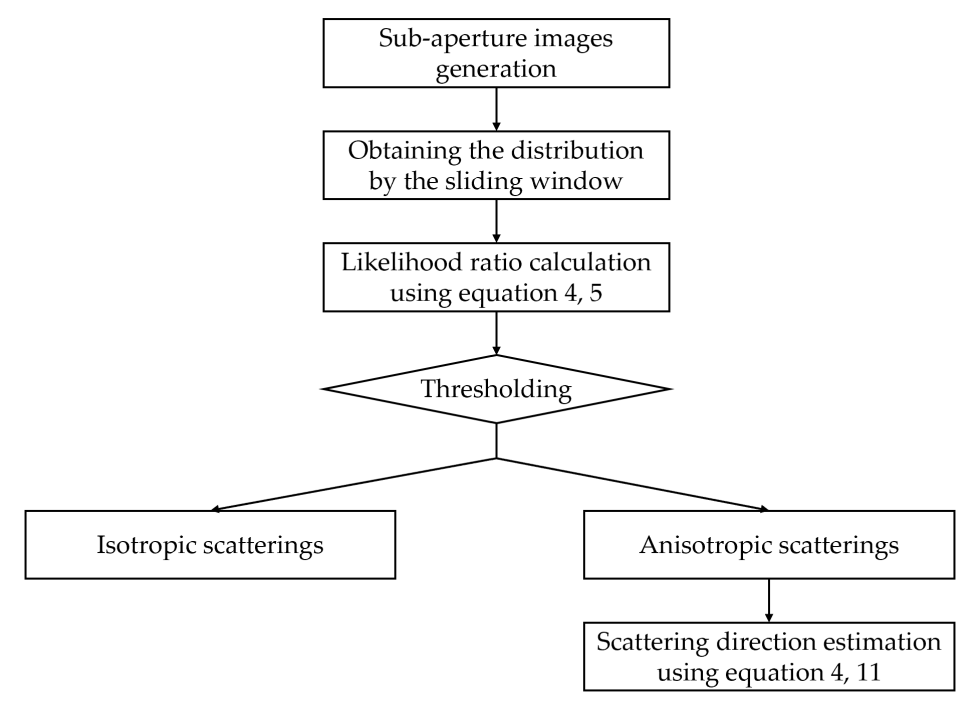

Figure 8. The flowchart of the anisotropic scattering analysis method.

The first step is sub-aperture images generation. The whole aperture is divided into $N$ sub-apertures with same length. There is a balance between the sub-aperture number $N$ and the sub-aperture length. If the length is too short, the azimuth resolution will be poor. If the length is too large, then the sub-aperture number is too small and the multi-angular scattering characteristic will be poor. It is adequate to choose the aperture length to make the azimuth resolution correspond to the range resolution. The range resolution can be calculated by Equation (6), where $c$ is the light velocity and $B$ is the bandwidth.

$$
\rho_{r}=\frac{c}{2 B}
$$

The azimuth resolution can be calculated by

$$
\rho_{a}=\frac{\lambda}{4 \sin \theta}
$$

$\lambda$ is the wavelength. $\theta$ is the angle corresponding to aperture length. Then sub-aperture images are obtained by using same imaging parameters. In this paper, data are all CSAR data. The focusing process is completed by using back-projection (BP) algorithm, which is the most popular imaging algorithm in CSAR imaging.

The second step is to obtain the distribution by using the sliding window. The sliding window moves pixel by pixel. The size of the window will influence both the accuracy of the parameter estimation and the spatial resolution of the result. The size of the sliding window will be discussed later. The distributions of the same pixel under different aspect angles can be obtained in every sub-aperture image. In this way, the multi-angular joint PDF of each pixel can be obtained. For $\mathrm{G}^{0}$ distribution [41] of the amplitude, the PDF is shown below.

$$
P(X)=\frac{-2 \alpha x}{\gamma\left(1+\frac{x^{2}}{\gamma}\right)^{1-\alpha}}, \text { where } \alpha, \gamma, x>0 .
$$

The parameters of $G^{0}$ distribution are very sensitive to the changes of the images [42]. The parameter $\alpha$ varies with the roughness of the image. The parameter $\gamma$ varies with the energy of the scattering. Therefore, $\mathrm{G}^{0}$ distribution can describe both homogeneous and heterogeneous regions in SAR images. For the region selected by the sliding window, pixels can be assumed to be independent from each other. The distributions under different aspect angles are independent too. For the selected region with $M$ pixels, there are $N$ sub-aperture images under $N$ aspect angles. 
The obtained distribution is assigned to the central pixel of the sliding window. The multi-angular joint PDF is

$$
P(X)=\prod_{k=1}^{N} \prod_{i=1}^{M} \frac{-2 \alpha_{k} x_{i k}}{\gamma_{k}\left(1+\frac{x_{i k}^{2}}{\gamma_{k}}\right)^{1-\alpha_{k}}}, \text { where } \alpha, \gamma, x>0 .
$$

The third step is likelihood ratio calculation. As can be seen in the equation, there are gamma functions in the expression. The non-linear degree of the PDF is very high so the parameter of $\mathrm{G}^{0}$ distribution cannot be estimated by maximum likelihood estimation (MLE), which is the common method of the parameter estimating. The parameter estimation of $G^{0}$ distribution has always been difficult since it was introduced. However, except the MLE, the parameters of $G^{0}$ distribution can be estimated by moment estimation [43]. When there are enough sampling points, the result of moment estimation is also very accurate. For $\mathrm{G}^{0}$ distribution of the amplitude, there are basically two kinds of moment estimation methods. One uses the second moment and fourth moment [44]; the other uses the 0.5 moment and first moment [41]. The second method will cause a large number of calculations. The likelihood ratio calculation is pixel-by-pixel and angle-by-angle so the computing method should be simple enough to be used in our method. Therefore, the first method was chosen to be used in our method. The moment estimations of $\alpha$ and $\gamma$ are calculated by

$$
\begin{aligned}
& \widehat{\alpha}=-1-\frac{m_{4}}{m_{4}-2 m_{2}^{2}}, \\
& \widehat{\gamma}=-m_{2}(\widehat{\alpha}+1) .
\end{aligned}
$$

After obtaining the distribution around each pixel under different aspect angles, the parameter can be estimated. For the numerator of the Equation (4), under the alternative hypothesis, the parameters are not all the same under different angles. The selected region can be expressed as $I_{k, p q} . p$ and $q$ is the row and column location of the central pixel. The parameters $\alpha_{k, p q}$ and $\gamma_{k, p q}$ can be estimated by $I_{k, p q}$ under $N$ aspect angles respectively. For the denominator of the Equation (4), the parameters are assumed all the same under different aspect angles. For a pixel, the distributions of its $N$ sub-aperture images selected by the sliding window share the same parameter. After collecting all the sub-aperture images $I_{k}$ together and making a new distribution, the parameters can be estimated. Combining Equation (9), the likelihood ratio can be calculated by putting the result of estimation into Equation (4).

After calculating the likelihood ratio in the vicinity of every pixel, a threshold value $\lambda_{t}$ can be set to get a binary image. There are detection procedures using constant false alarm rate (CFAR) to decide the threshold value [5,12]. However, the PDF of the likelihood ratio image of $G^{0}$ distribution is very complex after the likelihood ratio transformation. It is difficult to use CFAR. In addition, while using the CFAR, a high probability of detection comes from a high false alarm rate. To get a better result, the false alarm rate still needs to be set manually after testing even if CFAR is used. The threshold value optimization is not the focus of the proposed method. It can be easily obtained by machine learning using sufficient data in the future. Therefore, the experimental threshold value is used here in this paper. The value higher than the threshold means the scattering is anisotropic. In the opposite case, the scattering is isotropic. So far we have completed the anisotropic and isotropic scattering discrimination. Finally, there is the scattering direction estimation. The alternative hypothesis is changed into

$$
\begin{aligned}
H_{1}: \alpha_{j} & =\alpha_{0}, \alpha_{k}=\alpha_{00} \\
\gamma_{j} & =\gamma_{0}, \gamma_{k}=\gamma_{00}, \text { where } j, k=1,2, \cdots, N \text { and } k \neq j .
\end{aligned}
$$

$\alpha_{j}$ and $\gamma_{j}$ are the parameters under one of the aspect angles. $\alpha_{k}$ and $\gamma_{k}$ are the parameters under the rest of the angles. Under these angles, the distributions share the same parameters. $\alpha_{j, p q}$ and $\gamma_{j, p q}$ can 
be estimated using $I_{j, p q}$. For $\alpha_{k, p q}$ and $\gamma_{k, p q}$, the images $I_{k, p q}$ are combined together from $k=1$ to $k=N$ and $k \neq j$ and then the parameters are estimated. This means to assume that the anisotropic scattering exists under each angle in turn. If the scattering under $\theta_{k}$ is stronger than other angles, the likelihood ratio value of this angle should be the highest. The angle which has the strongest scattering is taken as the scattering direction. After doing the same operation for all anisotropic scattering, the scattering direction estimation is finished. At this point, the whole procedure of anisotropic scattering analysis method using $\mathrm{G}^{0}$ distribution is complete.

\subsection{Method Used for Comparison}

The likelihood ratio test method based on Wishart distribution using polarimetric SAR data [5] can be simplified to a single polarization version. Rayleigh distribution is the scalar version of the Wishart distribution. It is used to compare with the $\mathrm{G}^{0}$ distribution based methods proposed in this paper. Therefore, the anisotropic scattering analysis method using Rayleigh distribution is introduced briefly here. The steps are the same as the method using $\mathrm{G}^{0}$ distribution; only the parameter estimation method is different.

The multi-angular joint PDF of Rayleigh distribution is

$$
P(X)=\prod_{k=1}^{N} \prod_{i=1}^{M} \frac{x_{i k}}{\sigma_{k}^{2}} \exp \left(\frac{-x_{i k}^{2}}{2 \sigma_{k}^{2}}\right) .
$$

For Rayleigh distribution, the MLE of $\sigma^{2}$ is

$$
\sigma^{2}=\frac{1}{M} \sum_{i=1}^{M} x_{i}^{2}
$$

Under the null hypothesis, the MLE of $\sigma^{2}$ is

$$
\sigma_{0}^{2}=\frac{1}{M N} \sum_{k=1}^{N} \sum_{i=1}^{M} x_{i k}^{2}, \text { where } i=1,2, \cdots, M, k=1,2, \cdots, N .
$$

Under the alternative hypothesis, the MLE of $\sigma^{2}$ is

$$
\sigma_{k}^{2}=\frac{1}{M} \sum_{k=1}^{N} \sum_{i=1}^{M} x_{i k}^{2}, \text { where } i=1,2, \cdots, M, k=1,2, \cdots, N .
$$

According to Equations (4), (12), (14) and (15), the likelihood ratio can be expressed as

$$
\lambda=\left(\frac{\left(\frac{1}{N} \sum_{k=1}^{N} \eta_{k}\right)^{N}}{\prod_{k=1}^{N} \eta_{k}}\right)^{M}, \text { where } \eta_{k}=\frac{1}{M} \sum_{i=1}^{M} x_{i k}^{2} .
$$

Finally, the scattering direction of the anisotropic scattering can be estimated by

$$
\lambda_{j}=\left(\frac{\left(\eta_{j} \frac{1}{N-1} \sum_{k \neq j} \eta_{k}\right)^{N-1}}{\prod_{k=1}^{N} \eta_{k}}\right)^{M}, \text { where } \eta_{k}=\frac{1}{M} \sum_{i=1}^{M} x_{i k}^{2} .
$$

If $\lambda_{k}$ is the maximum value, $\theta_{k}$ is the scattering direction. 


\section{Experiment and Results}

In this section, the C-band airborne CSAR data are used to verify our anisotropic scattering analysis method. As can be seen in Figure 4, there are multiple types of targets in the scene. Anisotropic targets include buildings, storage tanks, street lamps, vehicles and so on. Isotropic targets include the lawn, bare land and roads. The results show that our method can successfully discriminate anisotropic scatterings from isotropic scatterings, and the scattering direction of the anisotropic scattering can be estimated. The result of using $\mathrm{G}^{0}$ distribution is much better than using Rayleigh distribution.

First, the anisotropic scattering analysis method using Rayleigh distribution was used on this data. According to the process introduced in Section 4, the first step is sub-aperture images generation. According to Equation (6), the range resolution of the radar is about $0.27 \mathrm{~m}$, and the sub-aperture length can be calculated by Equation (7). $3^{\circ}$ sub-aperture is a suitable value to make the azimuth resolution match the range resolution; 120 sub-aperture images were obtained by BP algorithm with same length and no gap. The size of each sub-aperture image is $1000 \times 1500$ pixels.

The second step is to obtain the distribution by the sliding window. A larger window helps with estimating the parameter but leads to a poor spatial resolution. A smaller window cannot estimate the parameter of the distribution well. To have a balance between the accuracy of the parameter estimation and the spatial resolution of the result, the size of the sliding window is discussed here. The relationship between the goodness-of-fit and the size of the sliding window was simulated by Monte Carlo method for both $\mathrm{G}^{0}$ distribution and Rayleigh distribution. In total, 100 groups of images obeying $\mathrm{G}^{0}$ distribution and Rayleigh distribution are generated. The parameters of these two distributions are generated randomly: $3 \times 3,5 \times 5,7 \times 7,9 \times 9,11 \times 11,13 \times 13$ window sizes are used in the simulation. $G^{0}$ distribution and Rayleigh distribution are used to fit the data selected by the window. The fitting result is compared with the pre-set PDF. The goodness-of-fit is still evaluated by adjusted R-square. Figure 9 shows the result. As can be seen in the figure, with the increase of the window size, the fitting result becomes better. In general, an adjusted R-square value higher than 0.95 indicates that the fitting result is good. Window size larger than $5 \times 5$ pixels can meet the requirement of the goodness-of-fit. Therefore, $5 \times 5$ pixels is chosen as the window size to get a better spatial resolution.

The next step is likelihood ratio calculation. The likelihood ratio can be calculated by Equation (16). The likelihood ratio image obtained by Rayleigh approach is shown in Figure 10. Lighter color denotes a higher likelihood ratio value while darker color denotes a lower value. As can be seen in the figure, the colors of the structure of the buildings, the street lamps and the storage tanks are lighter, which means they are anisotropic scatterings. The roads, the lawn, the concrete floor and the roofs of the buildings are dark in color. They all had isotropic scatterings. The results were as expected.

Then anisotropic scatterings can be discriminated from isotropic scatterings by thresholding. As mentioned before, experimental threshold value is used in this paper. After testing, $\lambda_{t}=215$ is suitable for the Rayleigh distribution-based method. Anisotropic scatterings are set to 1 while isotropic scatterings are set to 0 . The binary image after thresholding is shown in Figure 11. As can be seen in the figure, the anisotropic scattering can be discriminated from the isotropic scatterings. For example, there are buildings surrounded by the lawn and the concrete floor. In either case, the buildings are all segmented from the background.

The result of scattering direction estimation is shown in Figure 12. This is a color-coded direction image. The scattering direction corresponds to the flight trajectory. The colormap is shown in Figure 13. The result shows that the proposed method can estimate the scattering direction of the anisotropic scattering correctly. 


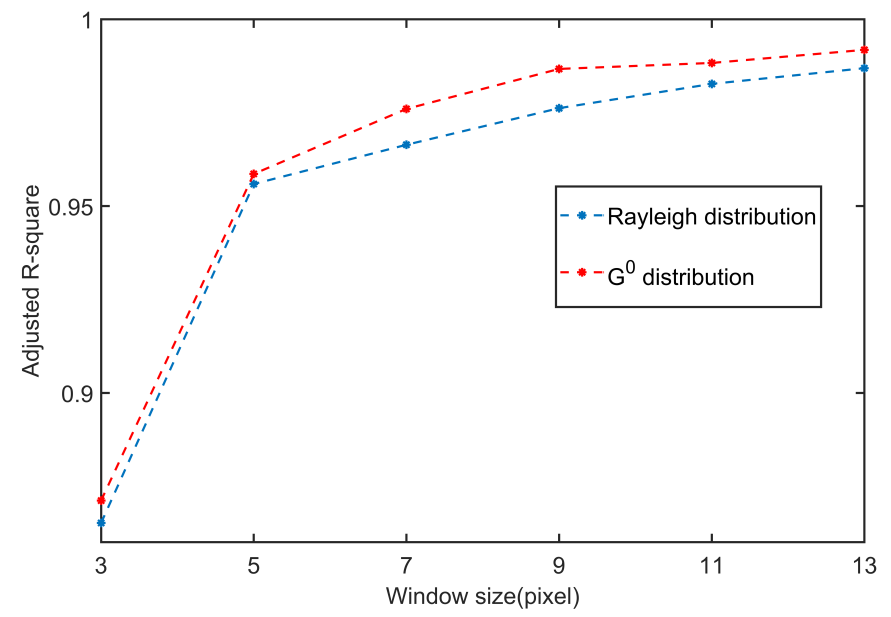

Figure 9. The relationship between the size of the sliding window and the goodness-of-fit.

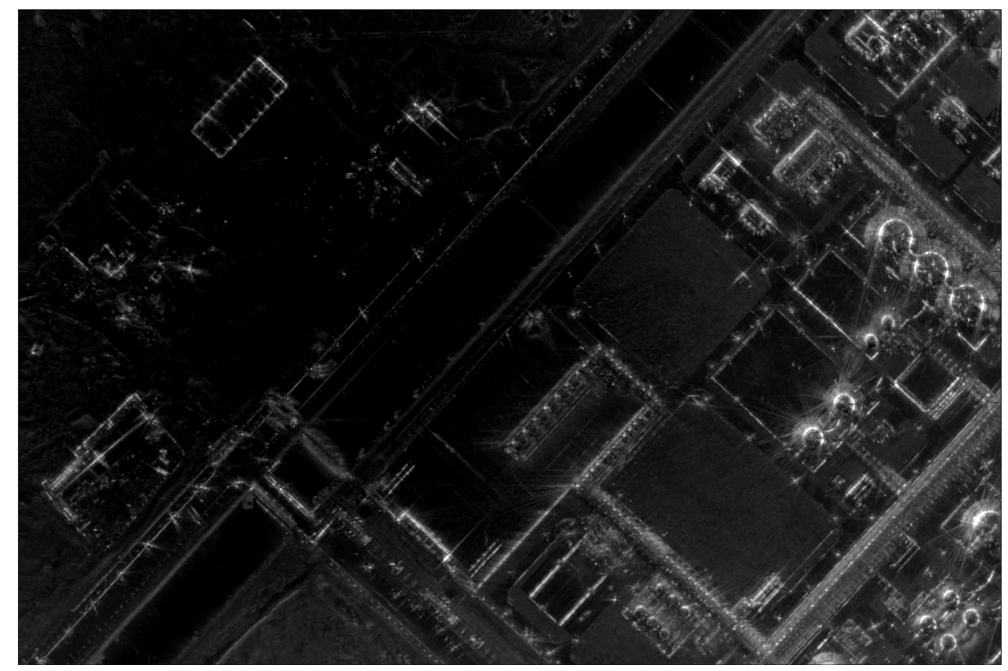

Figure 10. The likelihood ratio image obtained by Rayleigh approach.

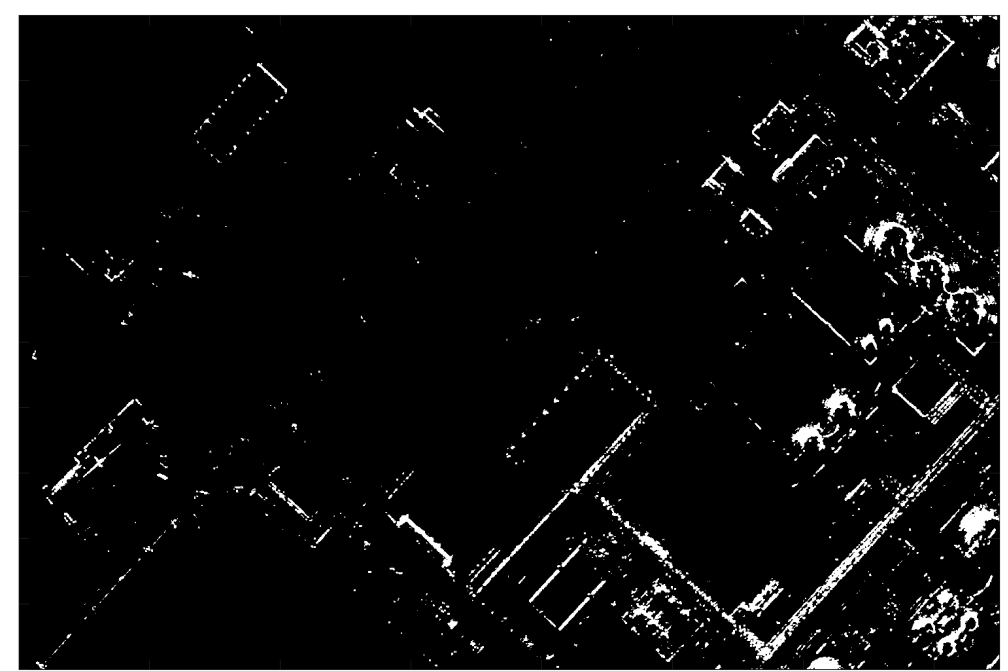

Figure 11. The binary image obtained by Rayleigh approach. 


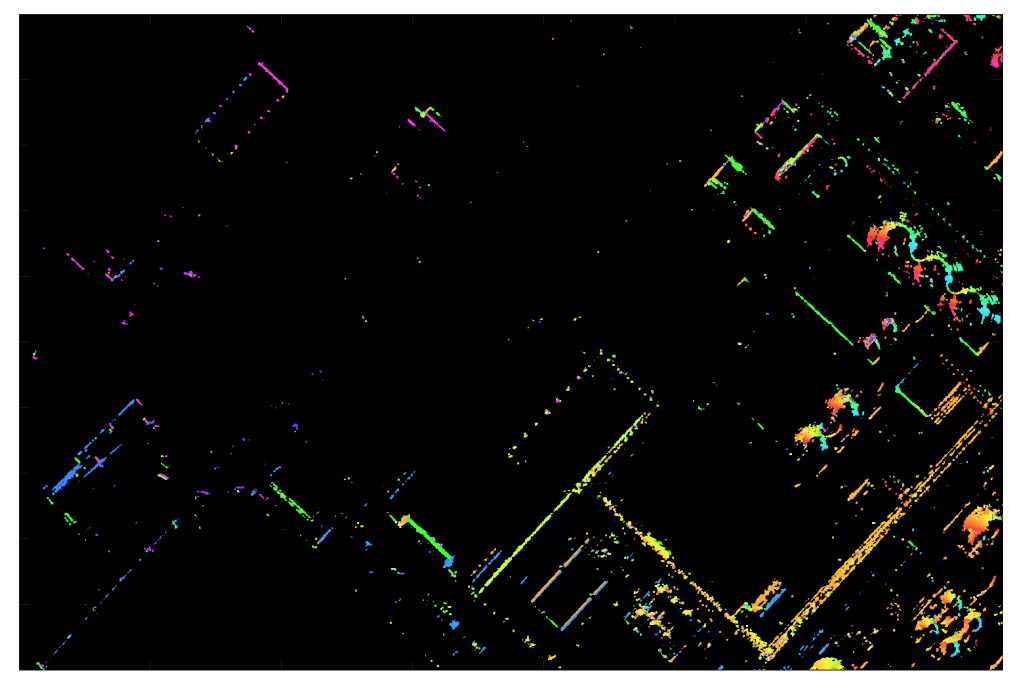

Figure 12. The scattering direction estimation result obtained by Rayleigh approach.

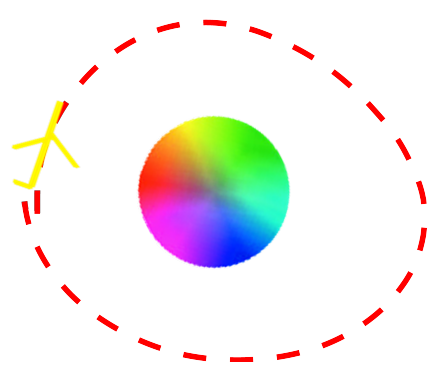

Figure 13. The colormap and the flight trajectory.

Then the method based on $\mathrm{G}^{0}$ distribution is used on the data. The sub-aperture length, imaging parameters and the size of the sliding window are all the same as in the Rayleigh approach. Therefore, the results using $\mathrm{G}^{0}$ distribution are given directly here. Figure 14 shows the likelihood ratio image. The contrast of the image is much stronger than the result of the Rayleigh approach. This is because $G^{0}$ distribution can better describe the distribution of the image than Rayleigh distribution. Better fitting effect means the parameters that estimated under different aspect angles are more accurate too. Therefore, the likelihood ratio value could be more accurate. An area consists of same scattering type can get a very similar likelihood ratio value. Rayleigh distribution cannot fit the distribution as well as $G^{0}$ distribution. The sliding window only contains 25 pixels. When there is a lot of noise under some aspect angles, the problem of poor fitting performance is more obvious and leads to a inaccurate likelihood ratio value.

The method using $\mathrm{G}^{0}$ distribution brings a more accurate likelihood ratio image, so the binary image should be better too. After testing, $\lambda_{t}=4.2 \times 10^{6}$ is suitable for the $\mathrm{G}^{0}$ distribution-based method. The binary image after thresholding is shown in Figure 15. Compared with Figure 11, the binary image obtained by the method using $\mathrm{G}^{0}$ distribution is much clearer than the result using Rayleigh distribution. There are almost no false alarms and misses in the results of the method using $\mathrm{G}^{0}$ distribution. Only anisotropic scatterings are white. The pixels around them are not judged as anisotropic scatterings. The comparison of local binary images between using Rayleigh distribution and $\mathrm{G}^{0}$ distribution is shown in Figure 16. The building area and the storage tanks are chosen as targets of interest. Comparing Figure 16a with Figure 16b, there are break points on the edge of the building in Figure 16a. Figure 16c shows the sub-aperture images under four aspect angles. As can be seen in the red circles, the whole edge of the building is anisotropic. When we use Rayleigh distribution, 
only scatterings with strong anisotropy are detected. The edge of the building is completely detected in the result of using $\mathrm{G}^{0}$ distribution. As can be seen in the red rectangles in Figure $16 \mathrm{~d}$,e, there are false alarms when we use Rayleigh distribution. Figure $16 \mathrm{f}$ shows that the area near the edge of the storage tank does not have anisotropy as strong as does the edge. The edge of the storage tank in the SAR image comes from the secondary scattering formed by the surface of the storage tank and the ground. It is strongly aspect-dependent. The area around the outside of the edge is the result of single scattering from the surface. This kind of scattering does not have strong directionality. Therefore, the proposed method using $\mathrm{G}^{0}$ distribution has a higher degree of distinction on the anisotropy of the scattering. This will be discussed more in Section 6.

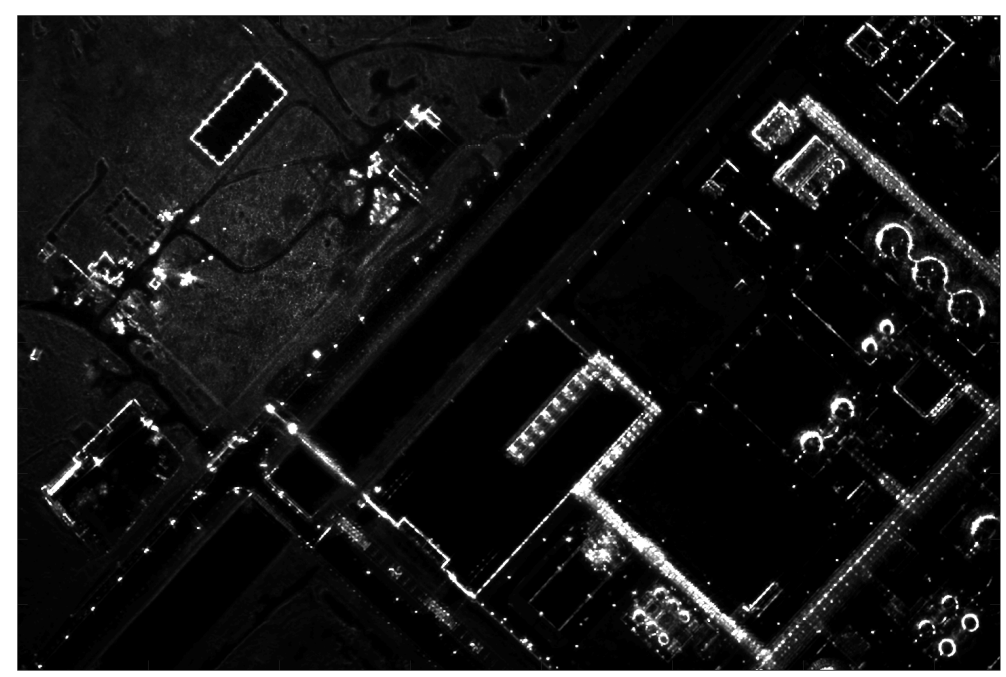

Figure 14. The likelihood ratio image obtained by $\mathrm{G}^{0}$ approach.

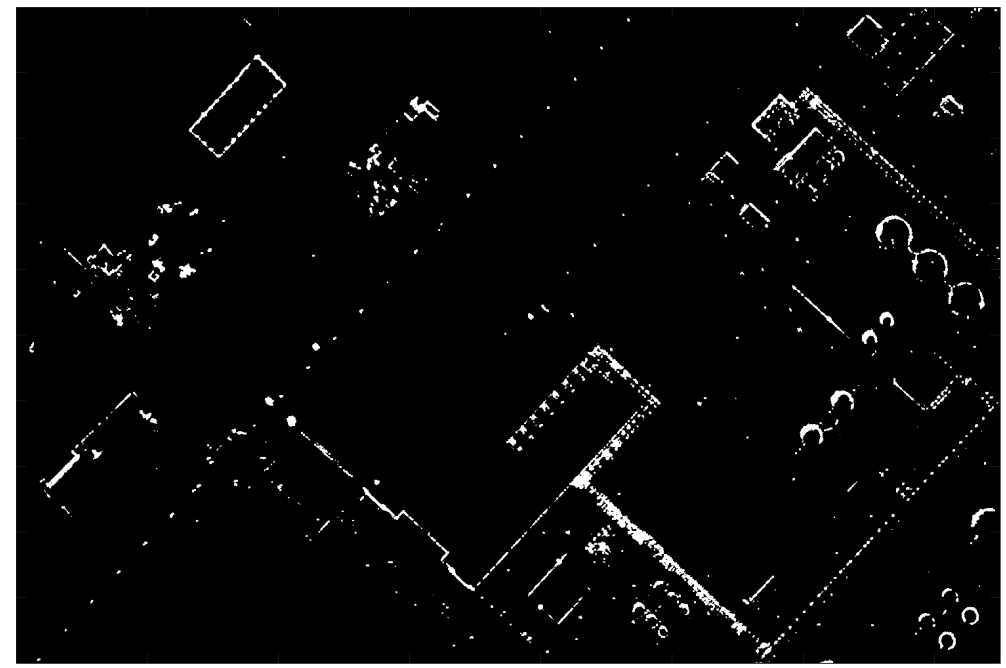

Figure 15. The binary image obtained by $\mathrm{G}^{0}$ approach.

The result of scattering direction estimation is shown in Figure 17. The colormap is the same as Figure 13. Benefiting from the better binary image, the scattering direction estimation result of the method using $\mathrm{G}^{0}$ distribution is clearer too. For each pixel, scattering directions estimated by using Rayleigh distribution and $\mathrm{G}^{0}$ distribution are basically all at the same aspect angles. They all accord to the fact. The scattering direction estimation can also help us to better understand the target and interpret the image. For example, the rectangular target in the upper left corner of the figure seems like a warehouse in the SAR image. Differently from other buildings in the scene, the scattering directions of the four sides are in the opposite direction that the wall facing. This means this is 
a wall structure without roof. According to the proposed scattering direction estimation method, the secondary scattering formed by the inner side of the wall and the ground is stronger than the secondary scattering formed by the outer side of the wall and the ground.

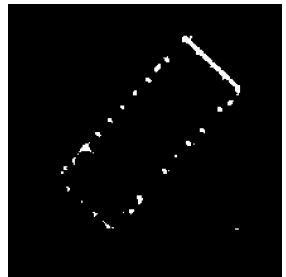

(a)

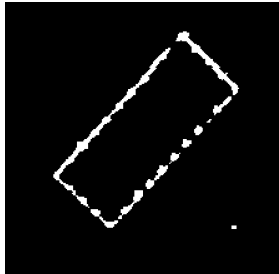

(b)
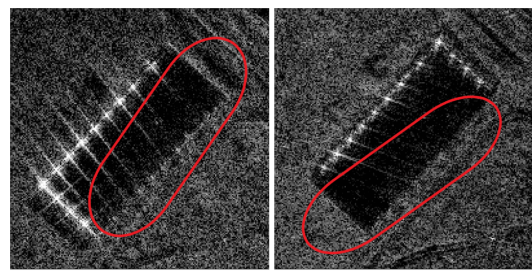

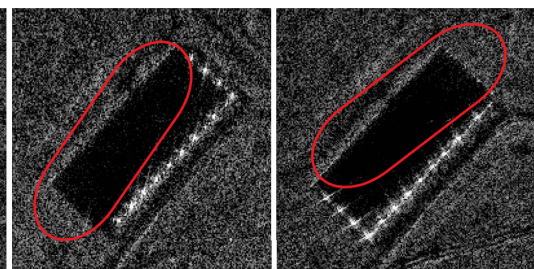

(c)

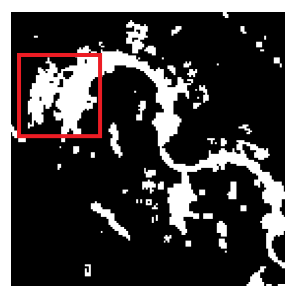

(d)
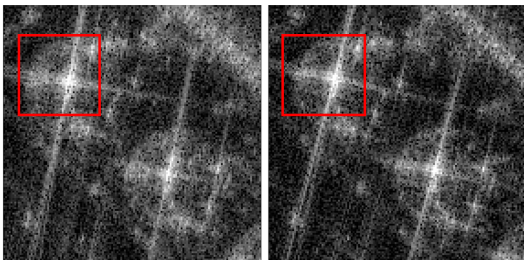

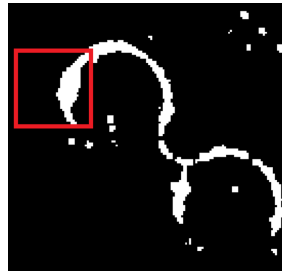

(e)
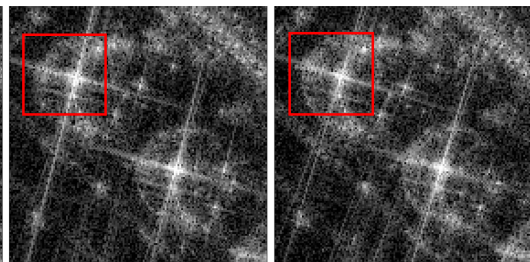

(f)

Figure 16. The comparison of the binary images by Rayleigh and $G^{0}$ approaches. (a) The building area of the Rayleigh approach. (b) The building area of $G^{0}$ approach. (c) The multi-angular images of the building area. (d) The storage tanks of the Rayleigh approach. (e) The storage tanks of $\mathrm{G}^{0}$ approach. (f) The multi-angular images of the storage tanks.

Through experimental verification, the proposed method can discriminate the anisotropic scattering and the isotropic scattering and estimate the scattering direction correctly. The likelihood ratio image of the method using $G^{0}$ distribution is much clearer than using Rayleigh distribution. The binary image of using $\mathrm{G}^{0}$ distribution has fewer false alarms and misdetections than using Rayleigh distribution. 


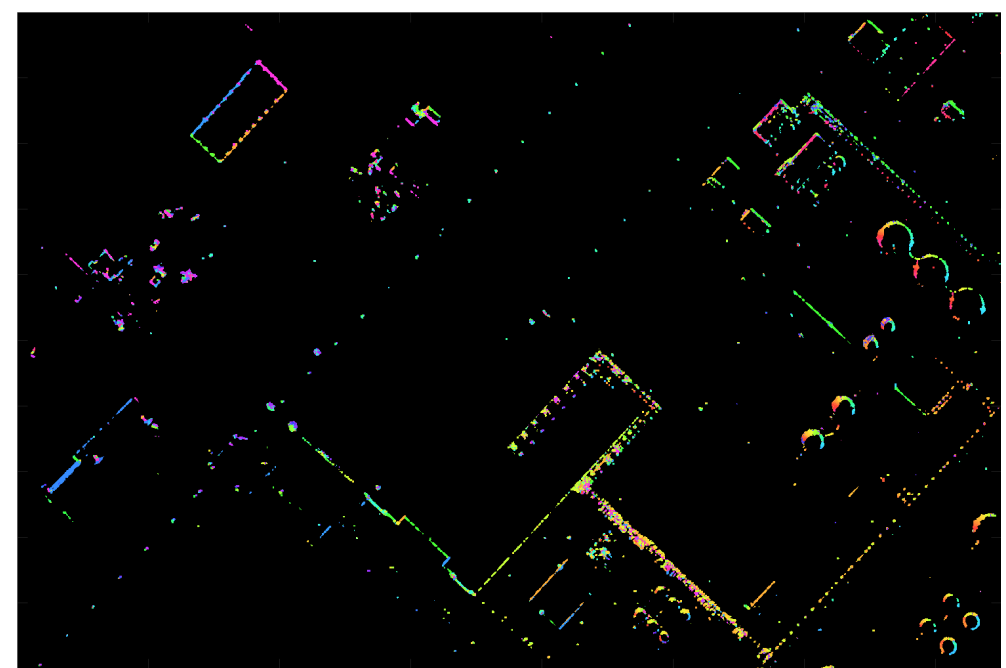

Figure 17. The scattering direction estimation result of the $G^{0}$ approach.

\section{Discussion}

An anisotropic scattering analysis method based on the multi-angular statistical properties is proposed in this paper. The likelihood ratio test is used to judge whether the scattering is anisotropic or isotropic. There is a method using the likelihood ratio test to detect the non-stationary scattering behavior [5]. They used the fully polarimetric SAR data at L-band. Polarization characteristics are very sensitive. Although the aspect look angle was $7.5^{\circ}$, an acceptable discrimination result was obtained. However, the azimuth beamwidth of the high-frequency radar is limited. The processing of full polarization SAR data is complex too. In addition, some radar systems only have the single polarization working mode. Therefore, the likelihood ratio test method is used on single polarization SAR data by us. A $G^{0}$ distribution based anisotropic scattering analysis method is proposed by us, using multi-angular single polarization SAR data. Rayleigh distribution is the scalar version of the original method proposed by Ferro-Famil et al. The result of using $\mathrm{G}^{0}$ distribution is much better than using Rayleigh distribution.

Comparing Figure 4 and Figure 15, it can be found that anisotropic targets usually have both strong scatterings and high levels of anisotropy. However, there are isotropic targets with strong scatterings and anisotropic targets with weak scatterings. Therefore, the anisotropic scattering analysis method should not be affected by the amplitude. In the proposed method, the likelihood ratio value indicates the stability of PDFs under different aspect angles. Anisotropic scatterings and isotropic scatterings can be discriminated by thresholding the likelihood ratio value. One may have the feeling that a similar result could be obtained by simply thresholding the SAR amplitude image. To clear up the doubt, the scatter density diagrams of pixels on amplitude-likelihood-ratio plane are shown in Figure 18. The x-axis is the amplitude of pixels and the $y$-axis is the normalized likelihood ratio value. The red line is the threshold value in our method. The amplitude and the likelihood ratio value have different discrimination capabilities. They are not linearly related. In the left part above the red line, these points indicate pixels with strong anisotropy and weak scatterings. These anisotropic scatterings cannot be discriminated from isotropic scatterings by the amplitude value. The purple triangles in Figure 18 indicate the pixels in the red rectangle in Figure 16d,e. It can be seen visually that the method using $\mathrm{G}^{0}$ distribution has higher a degree of distinction on the scattering anisotropy than using Rayleigh distribution. This is the reason why there were fewer false alarms in the result of using $\mathrm{G}^{0}$ distribution. The effectiveness and correctness of the proposed method can also be proved from this perspective. 


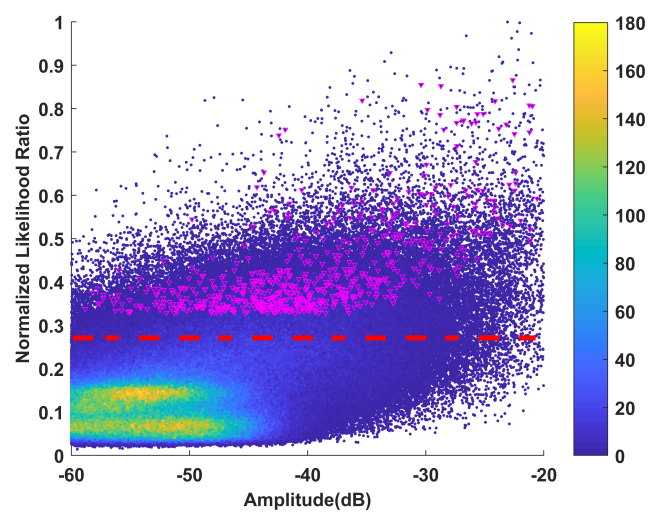

(a) Rayleigh approach.

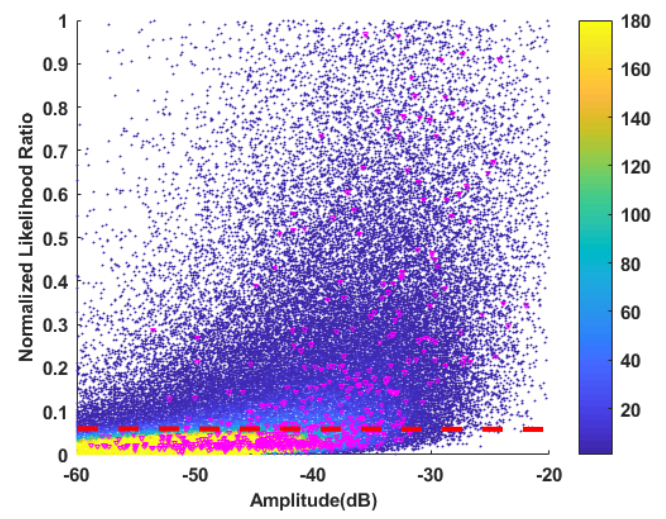

(b) $G^{0}$ approach.

Figure 18. The scatter density diagrams of pixels. (a) Rayleigh approach. (b) $\mathrm{G}^{0}$ approach.

For multi-angular SAR, especially with a very large aperture, the imaging result is affected by the elevation of the image plane. There will be an aspect-dependent geometrical distortion if the imaging height is different from the true height of the target. The result of anisotropic scattering analysis will be influenced. Therefore, the precise digital elevation model (DEM) should be obtained. Multi-angular SAR can extract the DEM of the scene using its own data. This has already been a necessary and mature step of the imaging procedure for us [22,45], so the proposed method can be used for an unknown area. Except the height error caused by the DEM model, 3D model of the complex target will lead to layovers too. This is not considered in this paper because we care more about the main characteristics of the target. All kinds of anisotropic scatterings are regarded as one kind. The aim of the proposed method is discriminating the strong aspect-dependent scatterings. The layover only exists under certain angles for a point. As shown in Figure 15, it would not really influence the result. This is also because the height of the building in the scene is low. It is very close to the DEM so the layover phenomenon is not obvious. Circular SAR can also extract the 3D model. In the future, a better result without layover can be obtained by using the 3D model of the scene.

Although the method was introduced and validated using circular SAR data, it can be used in all multi-angular SAR working modes. In engineering, the proposed method can provide automatically anisotropic scattering analysis results and handle large batches of tasks.

\section{Conclusions}

The statistical properties of anisotropic and isotropic scatterings were analyzed in this paper first. The statistical properties of the isotropic scattering are relatively stable under different aspect angles. The statistical properties of the anisotropic scattering change during the aspect angle variation. Second, the statistical model of the high-resolution multi-angular SAR images was studied. $\mathrm{G}^{0}$ distribution performs best in all kinds of areas and other distributions only perform well in specific areas. Then, an anisotropic scattering analysis method was proposed. It is based on the statistical property of multi-angular SAR images. A likelihood ratio test based on $\mathrm{G}^{0}$ distribution was used in the method to measure the scattering anisotropy. A C-band circular SAR data were used to validate our method. The result shows that the likelihood ratio value can describe the scattering anisotropy. Anisotropic scatterings can be discriminated from isotropic scatterings according to the likelihood ratio value. The scattering direction of the anisotropic scattering can be estimated too. The result of the proposed method is compared with the method using Rayleigh distribution, which is the scalar version of the original likelihood ratio test method using on fully polarimetric SAR data. The results of $\mathrm{G}^{0}$ distribution are better than Rayleigh distribution's. The proposed method can achieve the automated multi-angular SAR data processing and analysis in engineering. 
Author Contributions: Conceptualization, F.T.; funding acquisition, W.H.; investigation, F.T.; methodology, F.T.; resources, W.H.; supervision, Y.L., Y.W. and W.H.; validation, F.T., W.S. and S.F.; writing-original draft, F.T.; writing-review and editing, Y.L. and W.H. All authors have read and agreed to the published version of the manuscript.

Funding: This work was supported by the National Natural Science Foundation of China under grant 61571421, grant 61501210, grant 61431018 and grant 61331017.

Conflicts of Interest: The authors declare no conflict of interest.

\section{Reference}

1. Thompson, P.; Wahl, D.E.; Eichel, P.H.; Ghiglia, D.C.; Jakowatz, C.V.J. Spotlight-Mode Synthetic Aperture Radar: A Signal Processing Approach; Springer: Berlin/Heidelberg, Germany, 1996; Volume 26, pp. 330-332.

2. Munson, D.C.; Visentin, R.L. A signal processing view of strip-mapping synthetic aperture radar. IEEE Trans. Acoust. Speech Signal Process. 1989, 37, 2131-2147. [CrossRef]

3. Margarit, G.; Tabasco, A. Ship Classification in Single-Pol SAR Images Based on Fuzzy Logic. IEEE Trans. Geosci. Remote Sens. 2011, 49, 3129-3138. [CrossRef]

4. Duquenoy, M.; Ovarlez, J.P.; Ferro-Famil, L.; Pottier, E.; Vignaud, L. Characterization of scatterers by their anisotropic and dispersive behavior. In Proceedings of the IEEE International Geoscience \& Remote Sensing Symposium, Barcelona, Spain, 23-28 July 2007.

5. Ferro-Famil, L.; Reigber, A.; Pottier, E.; Boerner, W.M. Scene characterization using subaperture polarimetric sar data. IEEE Trans. Geosci. Remote Sens. 2003, 41, 2264-2276. [CrossRef]

6. Gherboudj, I.; Magagi, R.; Berg, A.A.; Toth, B. Soil moisture retrieval over agricultural fields from multi-polarized and multi-angular RADARSAT-2 SAR data. Remote Sens. Environ. 2011, 115, $33-43$. [CrossRef]

7. Schmitt, M.; Maksymiuk, O.; Magnard, C.; Stilla, U. Radargrammetric registration of airborne multi-aspect SAR data of urban areas. ISPRS J. Photogramm. Remote Sens. 2013, 86, 11-20. [CrossRef]

8. Ash, J.; Ertin, E.; Potter, L.C.; Zelnio, E. Wide-Angle Synthetic Aperture Radar Imaging: Models and algorithms for anisotropic scattering. Signal Process. Mag. IEEE 2014, 31, 16-26. [CrossRef]

9. Zhao, Y.; Hong, W.; Lin, Y.; Yu, L. Adaptive imaging of anisotropic target based on circular-SAR. Electron. Lett. 2016, 52, 1406-1408. [CrossRef]

10. Yue, Z.; Yun, L.; Ping, W.Y.; Wen, H.; Yu, L. Target multi-aspect scattering sensitivity feature extraction based on Circular-SAR. In Proceedings of the IGARSS 2016-2016 IEEE International Geoscience and Remote Sensing Symposium, Beijing, China, 10-15 July 2016.

11. Xue, F.; Lin, Y.; Hong, W.; Yin, Q.; Zhang, B.; Shen, W.; Zhao, Y. Analysis of Azimuthal Variations Using Multi-Aperture Polarimetric Entropy with Circular SAR Images. Remote Sens. 2018, 10, 123. [CrossRef]

12. Li, Y.; Qiang, Y.; Yun, L.; Wen, H. Anisotropy Scattering Detection From Multiaspect Signatures of Circular Polarimetric SAR. IEEE Geosci. Remote Sens. Lett. 2018, 15, 1575-1579. [CrossRef]

13. Barber, J. A Generalized Likelihood Ratio Test for Coherent Change Detection in Polarimetric SAR. IEEE Geosci. Remote Sens. Lett. 2015, 12, 1873-1877. [CrossRef]

14. Krylov, V.A.; Moser, G.; Voisin, A.; Serpico, S.B.; Zerubia, J. Change detection with synthetic aperture radar images by Wilcoxon statistic likelihood ratio test. In Proceedings of the ICIP International Conference on Image Processing, Orlando, FL, USA, 30 September-3 October 2012.

15. Xiong, B.; Chen, J.M.; Kuang, G. A change detection measure based on a likelihood ratio and statistical properties of SAR intensity images. Remote Sens. Lett. 2012, 3, 267-275. [CrossRef]

16. Schneider, R.Z.; Papathanassiou, K.; Hajnsek, I.; Moreira, A. Polarimetric and interferometric characterization of coherent scatterers in urban areas. IEEE Trans. Geosci. Remote Sens. 2006, 44, 971-984. [CrossRef]

17. D'Hondt, O.; Lopez-Martinez, C.; Ferro-Famil, L.; Pottier, E. Spatially Nonstationary Anisotropic Texture Analysis in SAR Images. IEEE Trans. Geosci. Remote Sens. 2008, 45, 3905-3918. [CrossRef]

18. Xue, F.T.; Yang, L.; Yun, L.; Qiang, Y.; Wen, H. An improved detection and feature retrieval method of anisotropic scattering for multi-aspect PolSAR data processing based on DRIA framework. In Proceedings of the 2016 IEEE International Geoscience and Remote Sensing Symposium (IGARSS), Beijing, China, 10-15 July 2016. 
19. Cantalloube, H.; Koeniguer, E.C. Assessment of physical limitations of High Resolution on targets at X-band from Circular SAR experiments. In Proceedings of the 7th European Conference on Synthetic Aperture Radar, Friedrichshafen, Germany, 2-5 June 2008.

20. Soumekh, M. Reconnaissance with slant plane circular SAR imaging. IEEE Trans. Image Process. A Publ. IEEE Signal Process. Soc. 1996, 5, 1252-1265. [CrossRef]

21. Chan, T.K.; Kuga, Y.; Ishimaru, A. Experimental studies on circular SAR imaging in clutter using angular correlation function technique. Geosci. Remote Sens. IEEE Trans. 1999, 37, 2192-2197. [CrossRef]

22. Yun, L.; Wen, H.; Tan, W.; Wang, Y.; Xiang, M. Airborne circular SAR imaging: Results at P-band. In Proceedings of the 2012 IEEE International Geoscience and Remote Sensing Symposium, Munich, Germany, 22-27 July 2012.

23. Pinheiro, M.; Prats, P.; Scheiber, R.; Nannini, M.; Reigber, A. Tomographic 3D Reconstruction From Airborne Circular SAR. In Proceedings of the 2009 IEEE International Geoscience and Remote Sensing Symposium, IGARSS 2009, Cape Town, South Africa, 12-17 July 2009.

24. Palm, S.; Oriot, H.M.; Cantalloube, H.M. Radargrammetric DEM Extraction Over Urban Area Using Circular SAR Imagery. IEEE Trans. Geosci. Remote Sens. 2012, 50, 4720-4725. [CrossRef]

25. Knott, E.F. Radar Cross Section Measurements. Proc. IEEE 2005, 75, 498-516.

26. Paladini, R.; Martorella, M.; Berizzi, F. Classification of Man-Made Targets via Invariant Coherency-Matrix Eigenvector Decomposition of Polarimetric SAR/ISAR Images. IEEE Trans. Geosci. Remote Sens. 2011, 49, 3022-3034. [CrossRef]

27. Duquenoy, M.; Ovarlez, J.P.; Vignaud, L.; Ferro-Famil, L.; Pottier, E. Classification based on the polarimetric dispersive and anisotropic behavior of scatterers. In Proceedings of the International Workshop on Science \& Applications of Sar Polarimetry \& Polarimetric Interferometry, Frascati, Italy, 22-26 January 2007; Volume 644.

28. Ulander, L.M.H.; Hellsten, H.; Stenstrom, G. Synthetic-aperture radar processing using fast factorized back-projection. IEEE Trans. Aerosp. Electron. Syst. 2003, 39, 760-776. [CrossRef]

29. Ponce, O.; Prats, P.; Rodriguez-Cassola, M.; Scheiber, R.; Reigber, A. Processing Of Circular SAR Trajectories With Fast Factorized Back-Projection. In Proceedings of the IEEE International Geoscience and Remote Sensing Symposium (IGARSS), Vancouver, BC, Canada, 24-29 July 2011.

30. Jakeman, E.; Pusey, P. A model for non-Rayleigh sea echo. Antennas Propag. IEEE Trans. 1976, $24,806-814$. [CrossRef]

31. Jao, J. Amplitude distribution of composite terrain radar clutter and the K-Distribution. IEEE Trans. Antennas Propag. 1984, 32, 1049-1062.

32. Lee, J.S.; Hoppel, K.; Mango, S.; Miller, A. Intensity and phase statistics of multilook polarimetric and interferometric SAR imagery. IEEE Trans. Geosci. Remote Sens. 1994, 32, 1017-1028.

33. Ulaby, F.T. Textural information in SAR images. IEEE Trans. Geosci. Remote Sens. 1986, 24, 235-245. [CrossRef]

34. Magee, L. R2 Measures Based on Wald and Likelihood Ratio Joint Significance Tests. Am. Stat. 1990, $44,250-253$.

35. Willmott, C.; Matsuura, K. Advantages of the mean absolute error (MAE) over the root mean square error (RMSE) in assessing average model performance. Clim. Res. 2005, 30, 79-82. [CrossRef]

36. Mittlböck, M.; Waldhör, T. Adjustments for R2-measures for Poisson regression models. Comput. Stat. Data Anal. 2000, 34, 461-472. [CrossRef]

37. Kuruoglu, E.E.; Zerubia, J. Modeling SAR Images With a Generalization of the Rayleigh Distribution. IEEE Trans. Image Process. 2004, 13, 527-533. [CrossRef]

38. Szajnowski, W.J. Estimators of Log-Normal Distribution Parameters. IEEE Trans. Aerosp. Electron. Syst. 1977, AES-13, 533-536. [CrossRef]

39. Fernandes, D. Segmentation of SAR images with Weibull distribution. In Proceedings of the 1998 IEEE International Geoscience and Remote Sensing Symposium Proceedings, Seattle, WA, USA, 6-10 July 1998.

40. Wang, H.; Ouchi, K. Accuracy of the K-Distribution Regression Model for Forest Biomass Estimation by High-Resolution Polarimetric SAR: Comparison of Model Estimation and Field Data. IEEE Trans. Geosci. Remote Sens. 2008, 46, 1058-1064. [CrossRef]

41. MLAFrery, A.C.; Muller, H.J. A model for extremely heterogeneous clutter. IEEE Trans. Geosci. Remote Sens. 1997, 35, 648-659.

42. Gambini, J.; Mejail, M.E.; Jacobo-Berlles, J.; Frery, A.C. Accuracy of edge detection methods with local information in speckled imagery. Stat. Comput. 2008, 18, 15-26. [CrossRef] 
43. Vasconcellos, K.L.P.; Frery, A.C.; Silva, L.B. Improving estimation in speckled imagery. Comput. Stat. 2005, 20, 503-519. [CrossRef]

44. Freitas, C.C.; Frery, A.C.; Correia, A.H. The polarimetric G distribution for SAR data analysis. Environmetrics 2005, 16, 13-31. [CrossRef]

45. Feng, S.; Lin, Y.; Wang, Y.; Yang, Y.; Shen, W.; Teng, F.; Hong, W. DEM Generation With a Scale Factor Using Multi-Aspect SAR Imagery Applying Radargrammetry. Remote Sens. 2020, 12, 556. [CrossRef]

(C) 2020 by the authors. Licensee MDPI, Basel, Switzerland. This article is an open access article distributed under the terms and conditions of the Creative Commons Attribution (CC BY) license (http:/ / creativecommons.org/licenses/by/4.0/). 